\title{
Associação entre exposição ao comportamento sedentário, indicadores de sono e fatores comportamentais em adolescentes
}

\author{
Association between exposure to sedentary behavior, sleep indicators and behavioral factors in \\ adolescents
}

Asociación entre exposición a conductas sedentarias, indicadores del sueño y factores conductuales en adolescentes

\author{
Isadora Fernanda de Freitas Cunha \\ ORCID: https://orcid.org/0000-0001-9669-9810 \\ Universidade Federal de Sergipe, Brasil \\ E-mail: isadora-fernanda@hotmail.com \\ Roberto Jerônimo dos Santos Silva \\ ORCID: https://orcid.org/0000-0002-4578-7666 \\ Universidade Federal de Sergipe, Brasil \\ E-mail: rjeronimoss@gmail.com \\ Davi Soares Santos Ribeiro \\ ORCID: https://orcid.org/0000-0001-9816-2566 \\ AGES Centro Universitário, Brasil \\ E-mail: profdavi@live.com \\ Edivaneide Oliveira Lima \\ ORCID: https://orcid.org/0000-0002-6639-7173 \\ Universidade Federal de Sergipe, Brasil \\ E-mail: eolimasaude@hotmail.com \\ Jamile Bedoia do Nascimento Santos \\ ORCID: https://orcid.org/0000-0002-3625-4438 \\ Universidade Federal de Sergipe, Brasil \\ E-mail: jamilebedoia@gmail.com \\ Lucas Souza Santos \\ ORCID: https://orcid.org/0000-0003-2172-4596 \\ Secretaria Municipal de Educação de Aracaju, Brasil \\ E-mail: lucas_souzza@hotmail.com \\ Aldemir Smith Menezes \\ ORCID: https://orcid.org/0000-0001-5718-6457 \\ Instituto Federal de Educação, Ciência e Tecnologia, Brasil \\ E-mail: aldemirsmith@yahoo.com.br
}

\begin{abstract}
Resumo
O objetivo do estudo foi analisar a associação entre exposição ao comportamento sedentário (ECS), indicadores de qualidade do sono e outros comportamentos de risco à saúde em adolescentes do estado de Sergipe, Brasil. Trata-se de um estudo epidemiológico com delineamento transversal com amostra representativa de 4139 escolares da rede pública de Sergipe. A coleta dos dados foi através do questionário Global Student Health Survey (GSHS, OMS). A variável desfecho analisada no estudo foi a (ECS), considerou-se expostos os adolescentes com período $\geq 3$ horas por dia. Foram selecionadas como variáveis independentes o consumo de álcool, uso de drogas, tabagismo, duração e autoavaliação do sono e como variáveis de controle sexo, idade e a renda média familiar. Para associação entre as variáveis foi utilizado a regressão logística binária. A análise revelou que as variáveis sexo, idade, renda média familiar, consumo de álcool, uso de drogas e duração do sono mostraram-se associadas a ECS após ajuste. Dormir oito horas ou menos por dia apresentou-se como fator de proteção para ECS. O estudo concluiu que ECS dos estudantes possui elevada prevalência e associação com fatores multivariados entre os adolescentes de Sergipe, assim fica evidente a necessidade de intervenções que visem a redução da ECS e outros comportamentos de risco à saúde.

Palavras-chave: Comportamento sedentário; Sono; Adolescentes.
\end{abstract}

\begin{abstract}
The aim of the study was to analyze the association between exposure to sedentary behavior (ECS), sleep quality indicators and other health risk behaviors in adolescents in the state of Sergipe, Brazil. This is a cross-sectional epidemiological study with a representative sample of 4139 public school students in Sergipe. Data collection was through the Global Student Health Survey (GSHS, WHO). The outcome variable analyzed in the study was (ECS),
\end{abstract}


considering exposed adolescents with a period $\geq 3$ hours a day. Alcohol consumption, drug use, smoking, sleep duration and self-assessment were selected as independent variables, and sex, age and mean family income as control variables. For the association between the variables, binary logistic regression was used. The analysis revealed that the variables sex, age, mean family income, alcohol consumption, drug use and sleep duration were associated with ECS after adjustment. Sleeping eight hours or less a day was shown to be a protective factor for ECS. The study concluded that ECS of students has a high prevalence and association with multivariate factors among adolescents in Sergipe, thus the need for interventions aimed at reducing ECS and other health risk behaviors is evident.

Keywords: Sedentary behavior; Sleep; Adolescents.

\section{Resumen}

El objetivo del estudio fue analizar la asociación entre la exposición a conductas sedentarias (ECS), indicadores de calidad del sueño y otras conductas de riesgo para la salud en adolescentes del estado de Sergipe, Brasil. Se trata de un estudio epidemiológico transversal con una muestra representativa de 4139 estudiantes de escuelas públicas de Sergipe. La recopilación de datos se realizó a través de la Encuesta mundial de salud estudiantil (GSHS, OMS). La variable de resultado analizada en el estudio fue (ECS), considerando adolescentes expuestos con un período $\geq 3$ horas diarias. Se seleccionaron como variables independientes el consumo de alcohol, el consumo de drogas, el tabaquismo, la duración del sueño y la autoevaluación, y como variables de control el sexo, la edad y el ingreso familiar medio. Para la asociación entre las variables se utilizó regresión logística binaria. El análisis reveló que las variables sexo, edad, ingreso familiar medio, consumo de alcohol, consumo de drogas y duración del sueño se asociaron con el ECS después del ajuste. Se demostró que dormir ocho horas o menos al día es un factor protector para la ECS. El estudio concluyó que el ECS de los estudiantes tiene una alta prevalencia y asociación con factores multivariantes entre los adolescentes de Sergipe, por lo que es evidente la necesidad de intervenciones dirigidas a reducir el ECS y otras conductas de riesgo para la salud.

Palabras clave: Conducta sedentaria; Sueño; Adolescentes.

\section{Introdução}

Mudanças no contexto socioeconômico tem impulsionado alterações de padrões comportamentais e o aumento de fatores de risco para desenvolvimento de patologias em jovens (Jalali-Farahani et al., 2016). Em esfera global, a maioria dos adolescentes não atendem as recomendações internacionais à prática de atividades físicas voltada a conservação de uma boa saúde (da Costa et al., 2019), cuja prevalência está em cerca de 81\% dos jovens, com idade entre 11 e 17 anos, que possuem baixos níveis de atividade física (Guthold et al., 2020). Por outro lado, evidências recentes elencam que os efeitos prejudiciais da exposição ao comportamento sedentário são mais acentuados em pessoas fisicamente inativa (Biswas et al., 2018; Katzmarzyk et al., 2019).

A Exposição ao Comportamento Sedentário (ECS), caracterizada pela exposição ao tempo de tela, está associada a alguns desfechos de saúde, como o risco aumentado para obesidade, atraso no desenvolvimento cognitivo, hipertensão arterial, problemas de comportamento social, aptidão física, além do prejuízo no desenvolvimento escolar (Carson et al., 2016; Tremblay et al., 2017; X. Y. Wu et al., 2017). Além disso, evidências destacam a associação entre a ECS e comportamentos de risco à saúde em adolescentes, como uso de álcool e outras drogas (Peltzer \& Pengpid, 2016) e tabagismo (Silva et al., 2020). Na mesma direção, investigações apontam a relação entre ECS e indicadores do sono, como a qualidade e a duração do sono (Souza Neto et al., 2021).

O sono tem sido cada vez mais reconhecido como um componente importante tanto na saúde mental quanto na saúde física. Durante a adolescência a duração do sono tende a ser curta, devido as mudanças físicas, hormonais, demandas escolares e sociais (Matricciani et al., 2017). Pesquisas mostram elevada prevalência da inatividade física, tempo sedentário e qualidade do sono em adolescentes (Hallal et al., 2012; Matricciani et al., 2012; Troiano et al., 2008). Porém, o que mais preocupa os pesquisadores é o aumento do risco de obesidade em crianças e adolescentes estar relacionado à duração inadequada do sono (Miller et al., 2018; Y. Wu et al., 2016).

As Diretrizes Canadense recomendam que a duração do sono para adolescentes seja de 8 a 10 horas/dia (Tremblay et al., 2016), entretanto, estudo destaca forte redução da duração do sono durante a adolescência, podendo estar diretamente 
Research, Society and Development, v. 11, n. 1, e46311125213, 2022

(CC BY 4.0) | ISSN 2525-3409 | DOI: http://dx.doi.org/10.33448/rsd-v11i1.25213

relacionado às demandas escolares e aos fatores biológicos (Chong et al., 2021). A literatura cientifica ainda mostra controversas relacionadas a associação entre ECS, comportamentos de risco à saúde (uso de álcool, drogas, tabagismo) e indicadores do sono em adolescentes. Neste sentido, o estudo objetivou analisar a associação entre exposição ao comportamento sedentário, indicadores de qualidade do sono e outros comportamentos de risco à saúde em adolescentes do estado de Sergipe, Brasil.

\section{Metodologia}

Este estudo foi uma análise secundária dos dados de um levantamento epidemiológico intitulado "Tendência secular sobre comportamentos de risco à saúde em adolescentes: Estudo CRiS_Adolescentes” desenvolvido pelo Grupo de pesquisa em Educação Física e Saúde do Instituto Federal de Educação, Ciência e Tecnologia de Sergipe (GPEFiS/IFS), em 2016. De natureza quantitativa (Pereira et al., 2018), esta pesquisa utilizou um delineamento transversal, com adolescentes estudantes do ensino médio, dos oito territórios geográficos do estado de Sergipe, Brasil.

A população alvo do estudo foi limitada por adolescentes de 14 a 19 anos de idade, de ambos os sexos, regularmente matriculados, nos períodos diurnos e noturnos, da Rede Estadual de Ensino. De acordo com dados disponíveis na Secretaria de Estado da Educação, em 2015, a população matriculada na rede foi de 62.933 alunos (IBGE, 2016).

O cálculo do tamanho amostral na análise da prevalência, foi considerado a proporção do tamanho da população do território, a prevalência estimada em 50\% como a maior esperada, o intervalo de confiança em $95 \%$, o erro tolerável de $5 \%$ (StatCal.Epiinfo). Além das informações descritas anteriormente, para análise de associação foram considerados o poder estatístico de $80 \%$ e Odds Ratio (OR) de 1,2. Para não perder a representatividade amostral, foram acrescentados $20 \%$ de escolares devido a motivos como: idade diferente dos critérios estabelecidos, recusa dos participantes, não responder a questões importantes como, sexo e idade.

Para a seleção da amostra utilizou-se o processo de amostragem estratificada em dois estágios: 1) Processo de amostragem estratificada proporcional ao território e conforme ao porte da unidade de ensino (1= até 199 escolares; $2=200$ a 499 escolares; $3=500$ ou mais alunos) (Soares Neto et al., 2013). Com intuito de que todos os territórios fossem contemplados com todos os portes das escolas, foi adotado como critério o sorteio de $25 \%$ das unidades de ensino do Estado (160 unidades), totalizando em 42 colégios; 2) Mediante a utilização do processo aleatório simples, as turmas foram selecionadas de acordo com série e turno da escola, considerando 20 alunos por turma.

Os critérios adotados para inclusão dos participantes na pesquisa foram: estar matriculado regularmente nas turmas do $1^{\circ}$ ao $3^{\circ}$ ano do ensino médio das escolas selecionadas; ter idade entre 14 e 19 anos; estar presente no momento da aplicação do instrumento; preencher adequadamente o questionário distribuído.

A participação dos adolescentes na pesquisa foi voluntária, adotando-se a utilização do termo negativo de consentimento. Inicialmente o Secretário de Estado da Educação, autorizando a participação das instituições na pesquisa $\mathrm{O}$ termo de Consentimento Livre e Esclarecido foi assinado pelos diretores das Diretorias Regionais de Educação, diretores das unidades de ensino, adolescentes com $\geq 18$ anos. Também foi utilizado o termo Negativo de Consentimento Livre e Esclarecido assinado pelos pais dos alunos com idade inferior a 18 anos e esses escolares assinaram o termo de Assentimento. Além do Secretário de Estado da Educação, autorizar a participação das instituições no estudo. Essa investigação foi aprovada pelo Comitê de Ética em Pesquisa com Seres Humanos (CEP/IFS) sob o número do protocolo № 1.522.876/2016.

A coleta de dados foi realizada em dia letivo, na própria sala de aula, o questionário foi aplicado por dois pesquisadores treinados, em cada turma, para auxiliar os adolescentes no preenchimento. O instrumento utilizado foi o questionário auto administrado adaptado do Global Student Health Survey, proposto pela Organização Mundial da Saúde - 
OMS, composto por duas seções.

A variável desfecho analisada foi a exposição ao comportamento sedentário (ECS), por meio da questão objetiva: “Em um dia típico, quanto tempo você gasta sentado, assistindo TV, jogando no computador, conversando com amigos, jogando cartas ou dominó?" Considerou-se expostos os adolescentes com período $\geq 3$ horas por dia (Khan et al., 2019; Uddin et al., 2019). As variáveis independentes analisadas foram: consumo de álcool, uso de drogas, tabagismo, duração do sono e auto avaliação do sono e como variáveis de controle sexo, idade e a renda média familiar. (Quadro 1)

Quadro 1. Matriz analítica do estudo.

\begin{tabular}{|c|c|c|}
\hline Variáveis & Questão objetiva - GSHS & Categorização \\
\hline Faixa etária & Qual sua idade em anos? & $\begin{array}{l}14-15 \text { anos } \\
16-17 \text { anos } \\
18-19 \text { anos }\end{array}$ \\
\hline Sexo & Qual o seu sexo? & $\begin{array}{l}\text { Masculino } \\
\text { Feminino }\end{array}$ \\
\hline Renda familiar & $\begin{array}{l}\text { Qual é a renda familiar mensal (Salário Mínimo }=R \$ \\
880,00) ?\end{array}$ & $\begin{array}{l}<1 \mathrm{SM} \\
1 \text { a } 2 \mathrm{SM} \\
>2 \mathrm{SM}\end{array}$ \\
\hline Consumo de álcool & Você consome bebida alcoólica? & $\begin{array}{l}\text { Exposto }=\text { Sim } \\
\text { Não exposto = Não }\end{array}$ \\
\hline Uso de Drogas & Você já usou drogas? & $\begin{array}{l}\text { Exposto }=\text { Sim } \\
\text { Não exposto = Não }\end{array}$ \\
\hline Tabagismo & $\begin{array}{l}\text { Você fuma cigarros, ou outras formas de tabaco (como } \\
\text { cigarro de palha, cachimbo ou cigarro de fumo de } \\
\text { rolo)? }\end{array}$ & $\begin{array}{l}\text { Exposto }=\text { Sim } \\
\text { Não exposto= Não }\end{array}$ \\
\hline Duração do Sono & $\begin{array}{l}\text { Em uma semana normal (em média), quantas horas } \\
\text { você dorme por dia? }\end{array}$ & $\begin{array}{l}0=\leq 8 \text { horas } \mathrm{p} / \text { dia } \\
1=10 \text { horas ou mais } \mathrm{p} / \mathrm{dia} \\
2=9 \text { horas } \mathrm{p} / \mathrm{dia}\end{array}$ \\
\hline Auto avaliação do sono & Como você avalia a qualidade do seu sono? & $\begin{array}{l}\text { Negativa = Ruim; regular } \\
\text { Positiva = Boa; muito boa; excelente }\end{array}$ \\
\hline
\end{tabular}

Legenda: ${ }^{1}$ Salário Mínimo. Fonte: Global School - Based Student Health Survey (OMS/GSHS). Fonte: Autores.

A análise descritiva foi realizada através do cálculo das frequências e intervalos de confiança (IC95\%) das variáveis de interesse. Para a análise de associação foi usada a regressão logística binária bruta e ajustada. As variáveis com p-valor $\leq 0,20$ na análise bruta foram mantidas para análise multivariável. O nível de significância adotado foi $\mathrm{p}<0,05$.

\section{Resultados}

O instrumento da pesquisa foi respondido por 4.721 adolescentes. Foram excluídos da amostra os jovens com idade inferior a 14 anos (12), com idade superior a 19 anos (549), os que não responderam questões como idade (06), sexo (12) e os 
que não preencheram mais de $50 \%$ das questões (03). A amostra final foi composta por 4139 . O maior número de adolescentes está na faixa etária de 16 e 17 anos de idade, do sexo feminino, com renda familiar até 1 salário mínimo está descrita na Tabela 1 .

Tabela 1. Características gerais da amostra. Sergipe, Brasil.

\begin{tabular}{|c|c|c|c|}
\hline Variáveis & $\mathrm{n}$ & $\%$ & IC $95 \%$ \\
\hline \multicolumn{4}{|l|}{ Idade } \\
\hline $14-15$ & 743 & 17,9 & $15,1-20,7$ \\
\hline $16-17$ & 2216 & 53,4 & $51,3-55,5$ \\
\hline $18-19$ & 1180 & 28,4 & $25,8-31,0$ \\
\hline \multicolumn{4}{|l|}{ Sexo } \\
\hline Feminino & 2392 & 57,6 & $55,6-59,6$ \\
\hline Masculino & 1759 & 42,4 & $40,1-44,7$ \\
\hline \multicolumn{4}{|l|}{ Renda familiar } \\
\hline Até $1 \mathrm{SM}^{1}$ & 2337 & 60,1 & $58,1-62,1$ \\
\hline $1-2 \mathrm{SM}^{1}$ & 1108 & 28,5 & $25,8-31,2$ \\
\hline$>2 \mathrm{SM}^{1}$ & 444 & 11,4 & $8,4-14,4$ \\
\hline \multicolumn{4}{|c|}{ Consumo de álcool } \\
\hline Não exposto & 2815 & 68,3 & $66,6-70,0$ \\
\hline Exposto & 1308 & 31,7 & $29,2-34,2$ \\
\hline \multicolumn{4}{|l|}{ Uso de drogas } \\
\hline Não exposto & 3822 & 95,7 & $95,1-96,3$ \\
\hline Exposto & 170 & 4,3 & $1,3-7,4$ \\
\hline \multicolumn{4}{|l|}{ Tabagismo } \\
\hline Não Exposto & 3510 & 94,5 & $93,8-95,3$ \\
\hline Exposto & 203 & 5,5 & $2,4-8,6$ \\
\hline \multicolumn{4}{|l|}{ Duração do sono } \\
\hline$\leq 8$ horas & 2645 & 64,4 & $62,6-66,2$ \\
\hline 9 horas & 900 & 21,9 & $19,2-24,6$ \\
\hline 10 horas ou mais & 563 & 13,7 & $10,9-16,5$ \\
\hline \multicolumn{4}{|c|}{ Auto avaliação do sono } \\
\hline Positiva & 2680 & 65,3 & $63,5-67,1$ \\
\hline Negativa & 1425 & 34,7 & $32,2-37,2$ \\
\hline \multicolumn{4}{|c|}{ Exposição ao comportamento sedentário } \\
\hline Não exposto & 2273 & 54,8 & $52,8-56,9$ \\
\hline Exposto & 1843 & 44,4 & $42,1-46,7$ \\
\hline
\end{tabular}

Legenda: ${ }^{1}$ Salário Mínimo. Fonte: Autores (2021). 
A Tabela 2 apresenta a prevalência da ECS de acordo com variáveis socioeconômicas e comportamentais em adolescentes. Verificou-se maior prevalência de adolescentes que relataram baixo consumo de álcool, uso de drogas, tabagismo e com a duração do sono de $\leq 8$ horas por dia.

Tabela 2. Prevalência da exposição ao comportamento sedentário de acordo com variáveis socioeconômicas e comportamentais em adolescentes.

\begin{tabular}{|c|c|c|c|}
\hline \multirow{2}{*}{ Variáveis } & $(\%)$ & $\mathrm{n}$ & $p$ \\
\hline & \multicolumn{3}{|l|}{ Exposto } \\
\hline \multicolumn{4}{|l|}{ Idade } \\
\hline $14-15$ & $(20,6)$ & 380 & \multirow{3}{*}{$<0,001$} \\
\hline $16-17$ & $(55,6)$ & 1024 & \\
\hline $18-19$ & $(23,7)$ & 436 & \\
\hline \multicolumn{4}{|l|}{ Sexo } \\
\hline Feminino & $(60,2)$ & 1109 & \multirow{2}{*}{0,003} \\
\hline Masculino & $(39,8)$ & 734 & \\
\hline \multicolumn{4}{|l|}{ Renda } \\
\hline Até $1 \mathrm{SM}^{1}$ & $(53,5)$ & 931 & \multirow{3}{*}{$<0,001$} \\
\hline $1-2 \mathrm{SM}^{1}$ & $(32,4)$ & 563 & \\
\hline$>2 \mathrm{SM}^{1}$ & $(14,1)$ & 246 & \\
\hline \multicolumn{4}{|c|}{ Consumo de álcool } \\
\hline Não exposto & $(66,5)$ & 1218 & \multirow{2}{*}{0,022} \\
\hline Exposto & $(33,5)$ & 614 & \\
\hline \multicolumn{4}{|l|}{ Uso de drogas } \\
\hline Não exposto & $(94,4)$ & 1678 & \multirow{2}{*}{$<0,001$} \\
\hline Exposto & $(5,6)$ & 100 & \\
\hline \multicolumn{4}{|l|}{ Tabagismo } \\
\hline Não Exposto & $(94,1)$ & 1563 & \multirow{2}{*}{0,244} \\
\hline Exposto & $(5,9)$ & 98 & \\
\hline \multicolumn{4}{|l|}{ Duração do sono } \\
\hline$\leq 8$ horas & $(60,1)$ & 1096 & \multirow{3}{*}{$<0,001$} \\
\hline 9 horas & $(24,4)$ & 445 & \\
\hline 10 horas ou mais & $(15,6)$ & 284 & \\
\hline \multicolumn{4}{|c|}{ Auto avaliação do sono } \\
\hline Positiva & $(65,2)$ & 1193 & \multirow[t]{2}{*}{0,853} \\
\hline Negativa & $(34,8)$ & 638 & \\
\hline
\end{tabular}


As variáveis consumo de álcool, uso de drogas e duração do sono mostraram-se associadas a ECS, após ajuste por sexo, idade e renda média familiar. O tabagismo e a auto avaliação do sono não mostraram associação (Tabela 3).

Os adolescentes que se apresentaram expostos ao consumo de álcool tiveram maior chance de exposição ao comportamento sedentário quando comparados com aqueles que não consomem bebida alcoólica. Quanto a duração do sono, os adolescentes que dormem $\leq 8$ horas por dia tem menos chances de exposição ao comportamento sedentário, em relação aos que dormem 9 horas por dia.

Tabela 3. Associação entre fatores comportamentais e sono com a exposição ao comportamento sedentário em adolescentes do estado de Sergipe, Brasil.

\begin{tabular}{|c|c|c|c|c|c|c|}
\hline \multirow{2}{*}{ Variáveis } & \multicolumn{4}{|c|}{ Bruta } & \multicolumn{2}{|c|}{ Ajustada } \\
\hline & $\mathrm{OR}^{\mathrm{a}}$ & $\mathrm{IC}^{\mathbf{b}}(95 \%)$ & $p$ & OR & IC $(95 \%)$ & $p$ \\
\hline \multicolumn{7}{|l|}{ Idade $^{1}$} \\
\hline $14-15$ & 1,77 & $1,47-2,14$ & \multirow[b]{2}{*}{$<0,001$} & 1,74 & $1,43-2,11$ & \multirow[b]{2}{*}{$<0,00$} \\
\hline $16-17$ & 1,46 & $1,27-1,69$ & & 1,47 & $1,26-1,71$ & \\
\hline $18-19$ & 1 & - & - & 1 & - & - \\
\hline \multicolumn{7}{|l|}{$\operatorname{Sexo}^{2}$} \\
\hline Masculino & 1 & - & - & 1 & - & - \\
\hline Feminino & 1,21 & $1,06-1,37$ & 0,003 & 1,25 & $1,10-1,43$ & 0,001 \\
\hline \multicolumn{7}{|l|}{ Renda $^{3}$} \\
\hline Até 1SM & 1 & - & - & 1 & - & - \\
\hline $1-2 \mathrm{SM}$ & 1,55 & $1,34-1,79$ & \multirow{2}{*}{$<0,001$} & 1,57 & $1,36-1,82$ & \multirow{2}{*}{$<0,00$} \\
\hline$>2 \mathrm{SM}$ & 1,89 & $1,54-2,32$ & & 1,92 & $1,55-2,36$ & \\
\hline \multicolumn{7}{|l|}{ Consumo de álcool $^{4}$} \\
\hline Não exposto & 1 & - & - & 1 & - & - \\
\hline Exposto & 1,17 & $1,02-1,33$ & 0,022 & 1,22 & $1,05-1,40$ & 0,007 \\
\hline \multicolumn{7}{|l|}{ Uso de drogas ${ }^{4}$} \\
\hline Não exposto & 1 & - & - & 1 & - & - \\
\hline Exposto & 1,85 & $1,35-2,54$ & $<0,001$ & 1,8 & $1,29-2,51$ & 0,001 \\
\hline \multicolumn{7}{|l|}{ Tabagismo } \\
\hline Não exposto & 1 & - & - & - & - & - \\
\hline Exposto & 1,18 & $0,89-1,57$ & 0,244 & - & - & - \\
\hline \multicolumn{7}{|l|}{ Duração do Sono $^{4}$} \\
\hline$\leq 8$ horas & 0,73 & $0,62-0,85$ & $<0,001$ & 0,77 & $0,66-0,91$ & $<0,001$ \\
\hline 9 horas & 1 & - & - & 1 & - & - \\
\hline 10 horas ou mais & 1,05 & $0,85-1,30$ & 0,663 & 1,12 & $0,90-1,40$ & \\
\hline \multicolumn{7}{|l|}{ Auto avaliação do sono } \\
\hline Positiva & 1 & - & - & - & - & - \\
\hline Negativa & 1,01 & $0,89-1,15$ & 0,853 & - & - & - \\
\hline
\end{tabular}

Legenda: ${ }^{\mathrm{a}}$ Odds Ratio; ${ }^{\mathrm{b}}$ Intervalo de Confiança; ${ }^{1}$ ajustado para sexo e renda; ${ }^{2}$ ajustado para idade e renda; ${ }^{3}$ ajustado para idade e sexo; ${ }^{4}$ ajustados para idade, sexo e renda. Fonte: Autores (2021). 


\section{Discussão}

O estudo apresentou como principal achado associação entre exposição ao comportamento sedentário, duração do sono, consumo de álcool e drogas ilícitas em adolescentes. De acordo com os resultados encontrados, os adolescentes do sexo feminino apresentaram maiores chances de ECS em relação aos do sexo masculino, como também os adolescentes expostos ao consumo de álcool e uso de drogas mostraram associação a ECS. Embora neste estudo não houve associação entre ECS e tabagismo, pesquisa realizada com escolares da Itália mostrou associação inversa entre tabagismo e ECS (Lazzeri et al., 2013). Além disso a duração de sono desses adolescentes esteve associada a ECS como fator de proteção.

Verificou-se que 44,4\% dos escolares participantes da pesquisa estavam expostos aos comportamentos sedentários por três ou mais horas, corroborando com investigações nacionais (Dias et al., 2014; R. W. Ferreira et al., 2016) e internacionais (Shen et al., 2020; Zhu et al., 2019). O crescente número de escolares ECS pode estar relacionado ao aumento do uso de smartphones, computadores e uso de internet (E. Z. Ferreira et al., 2020; Louragli et al., 2018; Mohamed \& Bernouss, 2020).

Adolescentes mais novos (14-15 anos) apresentaram chances superiores a ECS em relação aos com mais idade. Pesquisa realizada com escolares brasileiros não mostrou associação entre a idade e ECS (Lourenço et al., 2018), embora outra colaboraram com os achados desta investigação (de Lucena et al., 2015). A associação entre idade e ECS é divergente na literatura, sendo encontrado desfechos mostrando que adolescentes mais velhos apresentaram chances mais elevadas de ECS (Khan et al., 2019; Peltzer \& Pengpid, 2016).

Também foi observada associação entre ECS e maior renda familiar mensal dos participantes, resultados consistentes com outras investigações (de Lucena et al., 2015; Mohammed et al., 2020). Essa relação pode ser explicada devido ao fácil acesso à internet, mídias, aquisição de computadores, smartphones, favorável ao poder aquisitivo, que induzem a maior ECS (Mohammed et al., 2020; Ye et al., 2018).

Escolares do sexo feminino apresentaram alta prevalência (60,2 \%) para a ECS, consistente com a prevalência global, a qual mostrou que meninas tem mais chances de estarem ECS em relação aos meninos (Uddin et al., 2019), e com estudos anteriores (Bae et al., 2018; R. M. A. Silva et al., 2020). Observou-se nesta investigação que as moças dispõem maiores chances de ECS em relação aos escolares do sexo masculino. Este dado pode ser explicado devido as restrições das meninas de brincar ao ar livre, atividades diárias e lazer pouco ativo contribuindo para desigualdade de gênero na ECS (Atkin et al., 2013; R. W. Ferreira et al., 2016).

ECS associou-se com outros comportamentos de risco à saúde, como o consumo de álcool e o uso de drogas. Verificou-se que adolescentes expostos ao consumo de bebidas alcoólicas e que fazem uso de outras drogas apresentaram maiores chances de estarem ECS em relação aqueles que não estão expostos ao uso. Investigações anteriores obtiveram resultados semelhantes em relação a associação do consumo de álcool e ECS (R. M. A. Silva et al., 2020; Vancampfort et al., 2019).

Outras pesquisas apresentaram a associação entre consumo de álcool e outras drogas e a ECS (Peltzer \& Pengpid, 2016; Uddin et al., 2019), assim como, outro estudo desenvolvido na cidade Teresina/PI, o consumo de bebidas alcoólicas foi associado ao tempo de tela tanto na semana quanto no final de semana (Nery et al., 2020). Esta associação direta entre o consumo de álcool e ECS, pode estar interligada a contextos culturais e propagandas de televisão que encorajam os adolescentes a consumirem bebidas alcoólicas (Pedrosa et al., 2011).

Outra associação encontrada foi entre ECS e duração do sono, a variável independente apresentou-se como fator de proteção, ou seja, escolares que dormem 8 horas ou menos por dia tem menos chances de estarem ECS quando comparados aos que dormem 9 horas ou mais por dia. Pesquisa realizada com escolares, apresentou dados semelhantes, onde o tempo sentado e a duração do sono foram correlacionados negativamente (Felden et al., 2016), os resultados mostraram influência 
desfavorável do aumento do tempo sentado na duração do sono (Hale \& Guan, 2015; Tambalis et al., 2018). Embora há diretrizes que estabeleçam a duração adequada do sono, alguns autores enfatizam a necessidade individual da duração considerando aspectos socioculturais, genética, e diferentes resultados de saúde (Chaput et al., 2016; Matricciani et al., 2013).

Esse estudo apresenta algumas limitações, como a ausência de medidas objetivas das variáveis, pois os dados obtidos foram através do auto relato, o que pode haver confusão e/ou omissão de dados.

\section{Conclusão}

Diante dos achados, foi possível identificar que a prevalência da ECS em escolares do sexo feminino é maior comparado aos meninos e que houve associação entre ECS, consumo de álcool, uso de drogas e duração do sono. Dormir oito horas ou menos por dia esteve como fator de proteção em relação a ECS, dado esse que oscila na literatura. Os resultados do estudo remetem a necessidade de intervenções na redução da ECS de escolares, além de futuras pesquisas, utilizando medidas objetivas com o público alvo e esclarecer associações que ainda são controversas na literatura.

\section{Referências}

Atkin, A. J., Corder, K., Ekelund, U., Wijndaele, K., Griffin, S. J., \& van Sluijs, E. M. F. (2013). Determinants of Change in Children's Sedentary Time. PLoS ONE, 8(6), 1-9. https://doi.org/10.1371/journal.pone.0067627

Bae, K. N., Kim, H. R., Rhie, Y. J., Lee, K. H., \& Nam, H. K. (2018). Daily sitting time associated with the risk of metabolic syndrome in Korean adolescents. Journal of Pediatric Endocrinology and Metabolism, 31(1), 63-69. https://doi.org/10.1515/jpem-2017-0110

Biswas, A., Oh, P. I., Faulkner, G. E., Bonsignore, A., Pakosh, M. T., \& Alter, D. A. (2018). The energy expenditure benefits of reallocating sedentary time with physical activity: A systematic review and meta-analysis. Journal of Public Health (United Kingdom), 40(2), 295-303. https://doi.org/10.1093/pubmed/fdx062

Carson, V., Hunter, S., Kuzik, N., Gray, C. E., Poitras, V. J., Chaput, J. P., Saunders, T. J., Katzmarzyk, P. T., Okely, A. D., Connor Gorber, S., Kho, M. E., Sampson, M., Lee, H., \& Tremblay, M. S. (2016). Systematic review of sedentary behaviour and health indicators in school-aged children and youth: An update. Applied Physiology, Nutrition and Metabolism, 41(6), S240-S265. https://doi.org/10.1139/apnm-2015-0630

Chaput, J., Gray, C. E., Poitras, V. J., Carson, V., Gruber, R., Olds, T., Weiss, S. K., Gorber, S. C., Kho, M. E., Sampson, M., Belanger, K., Eryuzlu, S., Callender, L., \& Tremblay, M. S. (2016). Systematic review of the relationships between sleep duration and health indicators in school-aged children and youth. Appl. Physiol. Nutr. Metab, 41(June), S266-S282. https://doi.org/https://doi.org/10.1139/apnm-2015-0627

Chong, K. H., Parrish, A. M., Cliff, D. P., Dumuid, D., \& Okely, A. D. (2021). Changes in 24-hour movement behaviours during the transition from primary to secondary school among Australian children. European Journal of Sport Science, 0(0), 1-11. https://doi.org/10.1080/17461391.2021.1903562

Costa, B. G. G., da Silva, K. S., da Silveira, P. M., Berria, J., Machado, A. R., \& Petroski, E. L. (2019). The effect of an intervention on physical activity of moderate-and-vigorous intensity, and sedentary behavior during adolescents' time at school. Revista Brasileira de Epidemiologia, 22. https://doi.org/10.1590/1980-549720190065

Lucena, J. M. S., Cheng, L. A., Cavalcante, T. L. M., da Silva, V. A., \& de Farias Júnior, J. C. (2015). Prevalência de tempo excessivo de tela e fatores associados em adolescentes. Revista Paulista de Pediatria: Orgao Oficial Da Sociedade de Pediatria de Sao Paulo, 33 (4), 407-414. https://doi.org/10.1016/j.rpped.2015.04.001

Dias, P. J. P., Domingos, I. P., Ferreira, M. G., Muraro, A. P., Sichieri, R., \& Gonçalves-Silva, R. M. V. (2014). Prevalence and factors associated with sedentary behavior in adolescents. Revista de Saude Publica, 48(2), 266-274. https://doi.org/10.1590/S0034-8910.2014048004635

Felden, É. P. G., Filipin, D., Barbosa, D. G., Andrade, R. D., Meyer, C., Beltrame, T. S., \& Pelegrini, A. (2016). Adolescentes com sonolência diurna excessiva passam mais tempo em comportamento sedentário. Revista Brasileira de Medicina Do Esporte, 22(3), 186-190. https://doi.org/10.1590/1517869220162203147290

Ferreira, E. Z., Oliveira, A. M. N. de, Medeiros, S. P., Gomes, G. C., Cezar-Vaz, M. R., \& Ávila, J. A. de. (2020). A influência da internet na saúde biopsicossocial do adolescente: revisão integrativa. Revista Brasileira de Enfermagem, 73(2), 1-9. http://dx.doi.org/10.1590/0034-7167-2018-0766

Ferreira, R. W., Rombaldi, A. J., Ricardo, L. I. C., Hallal, P. C., \& Azevedo, M. R. (2016). Prevalência de comportamento sedentário de escolares e fatores associados. Revista Paulista de Pediatria, 34(1), 56-63. https://doi.org/10.1016/j.rpped.2015.06.005

Guthold, R., Stevens, G. A., Riley, L. M., \& Bull, F. C. (2020). Global trends in insufficient physical activity among adolescents: a pooled analysis of 298 population-based surveys with 1.6 million participants. The Lancet Child and Adolescent Health, 4(1), 23-35. https://doi.org/https://doi.org/10.1016/S23524642(19)30323-2

Hale, L., \& Guan, S. (2015). Screen time and sleep among school-aged children and adolescents: A systematic literature review. Sleep Medicine Reviews, 21, 50-58. https://doi.org/10.1016/j.smrv.2014.07.007 
Hallal, P. C., Andersen, L. B., Bull, F. C., Guthold, R., Haskell, W., Ekelund, U., Alkandari, J. R., Bauman, A. E., Blair, S. N., Brownson, R. C., Craig, C. L., Goenka, S., Heath, G. W., Inoue, S., Kahlmeier, S., Katzmarzyk, P. T., Kohl, H. W., Lambert, E. V., Lee, I. M., ... Wells, J. C. (2012). Global physical activity levels: Surveillance progress, pitfalls, and prospects. The Lancet, 380(9838), 247-257. https://doi.org/10.1016/S0140-6736(12)60646-1

IBGE. (2016). Pesquisa nacional de saúde do escolar : 2015. In Pesquisa nacional de saúde do escolar : 2015 / IBGE, Coordenação de População e Indicadores Sociais.

Jalali-Farahani, S., Amiri, P., \& Chin, Y. S. (2016). Are physical activity, sedentary behaviors and sleep duration associated with body mass index-for-age and health-related quality of life among high school boys and girls? Health and Quality of Life Outcomes, 14(1), 1-9. https://doi.org/10.1186/s12955-016-0434-6

Katzmarzyk, P. T., Powell, K. E., Jakicic, J. M., Troiano, R. P., Piercy, K., \& Tennant, B. (2019). Sedentary Behavior and Health: Update from the 2018 Physical Activity Guidelines Advisory Committee. Medicine \& Science in Sports \& Exercise, 51(6), 1227-1241. https://doi.org/10.1249/MSS.0000000000001935

Khan, A., Uddin, R., Lee, E. Y., \& Tremblay, M. S. (2019). Sitting time among adolescents across 26 Asia-Pacific countries: a population-based study. International Journal of Public Health, 64(8), 1129-1138. https://doi.org/10.1007/s00038-019-01282-5

Lazzeri, G., Azzolini, E., Pammolli, A., De Wet, D., \& Giacchi, M. . (2013). Correlation between physical activity and sedentary behavior with healthy and unhealthy behaviors in Italy and Tuscan region: A cross sectional study. Journal of Preventive Medicine and Hygiene, 54(1), 41-48.

Louragli, I., Ahami, A., Khadmaoui, A., Mammad, K., \& Lamrani, A. C. (2018). Evaluation of the Nomophobia'S Prevalence and Its Impact on School Performance Among Adolescents in Morocco. Problems of Psychology in the 21st Century, 12(2), 84-94. https://doi.org/10.33225/ppc/18.12.84

Lourenço, C. L. M., Zanetti, H. R., Amorim, P. R., Mota, J. A. P. S., \& Mendes, E. L. (2018). Comportamento sedentário em adolescentes : prevalência e fatores associados Sedentary behavior in adolescents : prevalence and associated factors. Revista Brasileira de Ciência e Movimento, 26(3), 23-32.

Matricciani, L., Bin, Y. S., Lallukka, T., Kronholm, E., Dumuid, D., Paquet, C., \& Olds, T. (2017). Past, present, and future: trends in sleep duration and implications for public health. Sleep Health, 3(5), 317-323. https://doi.org/10.1016/j.sleh.2017.07.006

Matricciani, L., Blunden, S., Rigney, G., Psych, B. A. H., Williams, M. T., \& Olds, T. S. (2013). Children's Sleep Needs. Sleep, 36(4), 527-534. https://doi.org/http://dx.doi.org/10.5665/sleep.2538

Matricciani, L., Olds, T., \& Petkov, J. (2012). In search of lost sleep: Secular trends in the sleep time of school-aged children and adolescents. Sleep Medicine Reviews, 16(3), 203-211. https://doi.org/10.1016/j.smrv.2011.03.005

Miller, M. A., Kruisbrink, M., Wallace, J., Ji, C., \& Cappuccio, F. P. (2018). Sleep duration and incidence of obesity in infants, children, and adolescents: a systematic review and meta-analysis of prospective studies. Sleep, 41(4), 1-19. https://doi.org/10.1093/sleep/zsy018

Mohamed, G., \& Bernouss, R. (2020). A cross-sectional study on Internet addiction among Moroccan high school students, its prevalence and association with poor scholastic performance. International Journal of Adolescence and Youth, 25(1), 479-490. https://doi.org/10.1080/02673843.2019.1674165

Mohammed, O. Y., Tesfahun, E., \& Mohammed, A. (2020). Magnitude of sedentary behavior and associated factors among secondary school adolescents in Debre Berhan town, Ethiopia. BMC Public Health, 20(1), 1-7. https://doi.org/10.1186/s12889-020-8187-x

Nery, S. S. S., De Souza, W. L., De Lucena, J. M. S., Dos Santos, J. C., Nunes, A. P., Guimaraes, M. C. F., \& De Freitas Brito, A. (2020). Health Risk Behaviors in adolescents: An association between Time of Television and alcohol use. Motricidade, 16(2), 184-195. https://doi.org/10.6063/motricidade. 18278

Pedrosa, A. A. da S., Camacho, L. A. B., Passos, S. R. L., \& de Oliveira, R. de V. C. (2011). Consumo de álcool entre estudantes universitários. Cadernos de Saude Publica, 27(8), 1611-1621. https://doi.org/10.1590/S0102-311X2011000800016

Peltzer, K., \& Pengpid, S. (2016). Leisure time physical inactivity and sedentary behaviour and lifestyle correlates among students aged 13-15 in the association of southeast asian nations (ASEAN) member states, 2007-2013. International Journal of Environmental Research and Public Health, 13(2), 1-15. https://doi.org/10.3390/ijerph13020217

Pereira, A., Shitsuka, D., Parreira, F., \& Shitsuka, R. (2018). Metodologia da Pesquisa Científica. In Santa Maria. Ed. UAB/NET/UFSM. https://repositorio.ufsm.br/bitstream/handle/1/15824/Lic_Computacao_Metodologia-Pesquisa-Cientifica.pdf?sequence=1. Acesso em: 28 março 2020 .

Shen, H., Yan, J., Hong, J. T., Clark, C., Yang, X. N., Liu, Y., \& Chen, S. T. (2020). Prevalence of physical activity and sedentary behavior among Chinese children and adolescents: Variations, gaps, and recommendations. International Journal of Environmental Research and Public Health, 17(9), 1-18. https://doi.org/10.3390/ijerph17093066

Silva, R. M. A., de Souza Andrade, A. C., Caiaffa, W. T., de Medeiros, D. S., \& Bezerra, V. M. (2020). National Adolescent School-based Health Survey PeNSE 2015: Sedentary behavior and its correlates. PLoS ONE, 15(1), 1-14. https://doi.org/10.1371/journal.pone.0228373

Soares Neto, J. J., Karino, C. A., Jesus, G. R. de, \& Andrade, D. F. de. (2013). A infraestrutura das escolas públicas brasileiras de pequeno porte. Revista Do Serviço Público, 64(3), 377-391. https://doi.org/10.21874/rsp.v64i3.129

Souza Neto, J. M. de, Costa, F. F. da, Barbosa, A. O., Prazeres Filho, A., Santos, E. V. O. Dos, \& Farias Júnior, J. C. de. (2021). Prática De Atividade Física, Tempo De Tela, Estado Nutricional E Sono Em Adolescentes No Nordeste Do Brasil. Revista Paulista de Pediatria : Orgao Oficial Da Sociedade de Pediatria de Sao Paulo, 39, e2019138. https://doi.org/http://dx.doi.org/10.1590/1984-0462/2021/39/2019138

Tambalis, K. D., Panagiotakos, D. B., Psarra, G., \& Sidossis, L. S. (2018). Insufficient sleep duration is associated with dietary habits, screen time, and obesity in children. Journal of Clinical Sleep Medicine, 14(10), 1689-1696. https://doi.org/10.5664/jcsm.7374 
Research, Society and Development, v. 11, n. 1, e46311125213, 2022

(CC BY 4.0) | ISSN 2525-3409 | DOI: http://dx.doi.org/10.33448/rsd-v11i1.25213

Tremblay, M. S., Aubert, S., Barnes, J. D., Saunders, T. J., Carson, V., Latimer-Cheung, A. E., Chastin, S. F. M., Altenburg, T. M., Chinapaw, M. J. M., Aminian, S., Arundell, L., Hinkley, T., Hnatiuk, J., Atkin, A. J., Belanger, K., Chaput, J. P., Gunnell, K., Larou che, R., Manyanga, T., ,.. Wondergem, R. (2017). Sedentary Behavior Research Network (SBRN) - Terminology Consensus Project process and outcome. International Journal of Behavioral Nutrition and Physical Activity, 14(1), 1-17. https://doi.org/10.1186/s12966-017-0525-8

Tremblay, M. S., Carson, V., Chaput, J. P., Connor Gorber, S., Dinh, T., Duggan, M., Faulkner, G., Gray, C. E., Grube, R., Janson, K., Janssen, I., Katzmarzyk, P. T., Kho, M. E., Latimer-Cheung, A. E., LeBlanc, C., Okely, A. D., Olds, T., Pate, R. R., Phillips, A., ... Zehr, L. (2016). Canadian 24-hour movement guidelines for children and youth: An integration of physical activity, sedentary behaviour, and sleep. Applied Physiology, Nutrition and Metabolism, 41(6), S311-S327. https://doi.org/10.1139/apnm-2016-0151

Troiano, R. P., Berrigan, D., Dodd, K. W., Mâsse, L. C., Tilert, T., \& Mcdowell, M. (2008). Physical activity in the United States measured by accelerometer. Medicine and Science in Sports and Exercise, 40(1), 181-188. https://doi.org/10.1249/mss.0b013e31815a51b3

Uddin, R., Lee, E. Y., Khan, S. R., Tremblay, M. S., \& Khan, A. (2019). Clustering of lifestyle risk factors for non-communicable diseases in 304,779 adolescents from 89 countries: A global perspective. Preventive Medicine, 131, 105955. https://doi.org/10.1016/j.ypmed.2019.105955

Vancampfort, D., van Damme, T., Firth, J., Hallgren, M., Smith, L., Stubbs, B., Rosenbaum, S., \& Koyanagi, A. (2019). Correlates of leisure-time sedentary behavior among 181,793 adolescents aged 12-15 years from 66 low- And middle-income countries. PLoS ONE, 14(11), 1-14. https://doi.org/10.1371/journal.pone.0224339

Wu, X. Y., Han, L. H., Zhang, J. H., Luo, S., Hu, J. W., \& Sun, K. (2017). The influence of physical activity, sedentary behavior on health-related quality of life among the general population of children and adolescents: A systematic review. PLOS ONE, 12(11), e0187668. https://doi.org/10.1371/journal.pone.0187668

Wu, Y., Gong, Q., Zou, Z., Li, H., \& Zhang, X. (2016). Short sleep duration and obesity among children: A systematic review and meta-analysis of prospective studies. Obesity Research and Clinical Practice, 11(2), 140-150. https://doi.org/10.1016/j.orcp.2016.05.005

Ye, S., Chen, L., Wang, Q., \& Li, Q. (2018). Correlates of screen time among 8-19-year-old students in China. BMC Public Health, 18(1), 1-7. https://doi.org/10.1186/s12889-018-5355-3

Zhu, Z., Tang, Y., Zhuang, J., Liu, Y., Wu, X., Cai, Y., Wang, L., Cao, Z. B., \& Chen, P. (2019). Physical activity, screen viewing time, and overweight/obesity among Chinese children and adolescents: An update from the 2017 physical activity and fitness in China - The youth study. BMC Public Health, 19(1), 1-8. https://doi.org/10.1186/s12889-019-6515-9 